\title{
Non-sinusoidal wall oscillation for drag reduction
}

\author{
A. Cimarelli ${ }^{1}$, B. Frohnapfel ${ }^{2}$, Y. Hasegawa ${ }^{2,3}$, E. De Angelis ${ }^{1}$ and \\ M. Quadrio ${ }^{4},{ }^{1}$ University of Bologna, DIEM; ${ }^{2}$ Technical University \\ of Darmstadt, CSI; ${ }^{3}$ University of Tokyo; ${ }^{4}$ Politecnico di Milano;
}

The control of wall-bounded turbulent flows with the aim of reducing the wall-shear stress is an important and challenging topic in modern fluid mechanics. Indeed, such a reduction has very important beneficial effects for engineering flow systems. One prominent control technique which allows to achieve a significant reduction of turbulent drag is based on the modification of wall turbulence by the cyclic spanwise movement of the wall, [1]. Despite the studies available in the literature, there are a number of issues related to drag reduction properties of the oscillating wall that have not yet received a definite answer. For example, it is well known that the frequency and the amplitude of the oscillation play a first-order role in determining the amount of the turbulent drag reduction rate but the effects of the shapes of the wall oscillations are still not determined. Indeed, all the attemps carried out towards achieving a reduction of turbulent friction have used a sinusoidal shape for the spanwise oscillation of the wall. For that reasons, we investigate the drag reducing properties of non-sinusoidal wall oscillations. The present work aims at answering two related questions:

- How do deviations from the sinusoidal wave shape - which might occur when trying to realize an oscillating wall in practice - influence the control performance?

- Is it possible to obtain flow control performance superior to the sinusoidal wall oscillations with other oscillations shapes?

The analysis of the non-sinusoidal wall oscillations is performed using direct numerical simulation data of a turbulent channel flow. The simulations are carried out with an highly accurate numerical code. The friction Reynolds number of the reference simulation without wall oscillation is $R e_{\tau}=200$ and the computational domain is $6.67 h \times 2 h \times 3.33 h$. All simulations for the different boundary conditions of spanwise velocity at the walls are performed starting from the same initial condition of fully developed channel flow without wall oscillation.

The obtained results illustrate the dependency of the drag reduction rate, $R$, and of the power spent to move the walls, $P_{i n}$, with respect the shape of the wall motion. An analytical model based on the generalization of the laminar solution of the Stokes layer is derived which allows the a priori prediction of $R$ and $P_{i n}$ for different wall motions in turbulent flows under certain constraints.

[1] M. Quadrio, Drag reduction in turbulent boundary layers by in-plane wall motion, Phil. Trans. R. Soc. A 369 (2011), $1428-1442$. 